\title{
DRIVING PERFORMANCE IN A SIMULATOR AS A FUNCTION OF PAVEMENT AND SHOULDER WIDTH, EDGE LINE PRESENCE, AND ONCOMING TRAFFIC
}

\author{
Susan T. Chrysler, Alicia A. Williams \\ Human Factors Program \\ Center for Transportation Safety \\ Texas Transportation Institute \\ Texas A\&M University System \\ College Station, Texas, USA \\ E-mail: s-chrysler@tamu.edu \\ E-mail: a-williams@ttimail.tamu.edu
}

\begin{abstract}
Summary: Driving simulation has primarily been used to study issues of driver distraction and to evaluate in-vehicle devices. The visualization and driver performance capabilities of simulators can be applied to more traditional traffic engineering problems as well. This project aims to demonstrate the usefulness of a driving simulator in evaluating geometric designs for two-lane roads. Paved surface width has been shown to be correlated with crash rates and travel speeds on two-lane rural roads throughout Texas. The current project examines how travel lane width, edge line striping, and shoulder width affect driver errors on these roadway types. Issues of simulator validity, scenario development, and simulator sickness are discussed.
\end{abstract}

\section{RURAL TWO-LANE ROAD CRASHES}

Within Texas' 74,000 miles of state system roadway, 52,000 miles are classified as secondary roads. Of these roadways, $88 \%$ of State Highways are two-lane roads and 99\% of Farm-toMarket roads are two-lane. An examination of the year 2000 crash data shows that Texas ranks highest nationally in rural traffic fatalities and eighth highest in the rural traffic fatality rate. In Texas, the fatal crash rate on two-lane roads is between 2-5 times higher than on Interstate highways (Mounce, 2004). A large portion of these crashes on two-lane rural roads are singlevehicle run-off-the-road crashes. A preliminary crash analysis has revealed more fatal and serious injury crashes on roadways with paved surface widths of 20 feet or less when compared with widths of 24 feet or greater. Other differences exist between these two types of roadways, such as edge line striping, shoulder width, and restricted right of way. While the crash data show a relationship, the exact nature of the driver error leading to crashes is not known. Hauer (2000) explains the benefits of wider lanes as providing more room for correction in near-accident circumstances and also increasing the separation between vehicles in adjacent lanes. Although he states the pitfall is the tendency for traffic to travel at faster speeds on wider lanes.

A driving simulator study was conducted to examine driver behavior in tangent and curve sections of roadways with various travel lane and shoulder widths. The reader is referred to the full technical report for results and additional details of the study (Chrysler, Williams, and Meischen, 2005). 


\section{EXPERIMENTAL DESIGN}

\section{Driving Simulator}

Data collection was conducted in Texas Transportation Institute's DriveSafety ${ }^{\text {TM }}$ Version 1.6.2 fixed-base driving simulator. The vehicle, a complete and full-size 1995 Saturn SL automobile, is outfitted with computers, potentiometers, and torque motors connected to the accelerator, brakes, and steering. The windshield of the vehicle has been removed. Computer-generated driving scenes are sent to three high-resolution projectors and projected to three high-reflectance screens producing a 150-degree field of view.

\section{Tile Development}

Based on the crash findings previously mentioned, eight different test roadway geometries were developed. These eight conditions were submitted to a software programming contractor for development to interface with our existing DriveSafety ${ }^{\mathrm{TM}}$ software used by the simulator. All scenes were daytime clear conditions. From each condition, 2 roadway segments, or tiles, would be developed: a $200 \mathrm{~m}$ straightaway or tangent, and a $600 \mathrm{~m}$ segment consisting of a curve to the right, and then the same curve to the left.

Pavement Characteristics. A pavement surface with the appearance of asphalt was used for both the roadway and shoulder textures. Beyond the edge of the shoulder was a jagged dirt area, and then finally grass fields (see Figure 1). Whenever an edge line was present, the edge line took up 4" of roadway space and was white. Along the curved section of roadways, solid double centerline striping was present, and along the tangent sections, single dashed striping was present to represent allowed passing maneuvers. The new tiles were not designed to provide tactile feedback when the simulator vehicle crosses the center or edge line; however, depending on the speed and angle of the vehicle, some feedback in the steering wheel produced by a torque motor may have been experienced if the vehicle left the shoulder pavement.

Horizontal Curve Segment Specifications. As mentioned above, the curved segments have nopassing centerline markings. Each curve tile has one 3-degree horizontal curve with a 2,000 foot radius to the right, and then the identical curve to the left. The curve super elevation is 6 degrees, with the banking beginning prior to the entry into the curve and terminating after the curve. The total length of each curve tile, with a curve in each direction, is $600 \mathrm{~m}$.

Roadside Environment. The roadside environment for the test conditions included occasional fields, pastures, clusters of trees, and even fenced-in areas with livestock. During the filler segments, the environment is constantly changing to include houses and other buildings found in rural settings. The filler segments also contain various types of intersections with appropriate signage. Each of the tiles for the test conditions had identical surroundings to avoid introducing another variable that might affect the subjects' lane position. Also, no driveways, intersections, or signs were added to the testing tiles. 


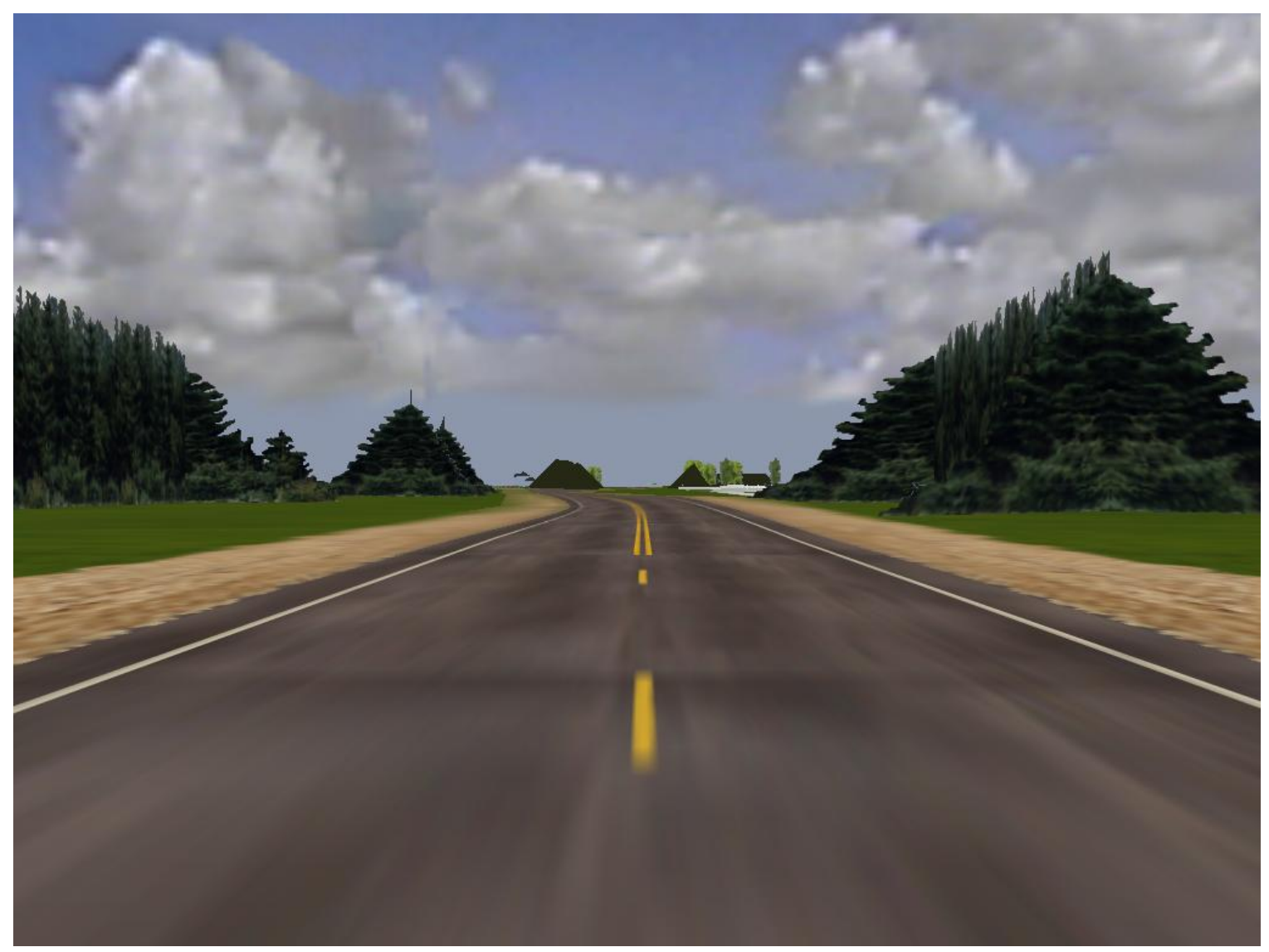

Figure 1. Simulator Pavement and Shoulder Textures

\section{Experimental Procedure}

Participants. Thirty-six volunteer drivers, consisting of 9 women and 27 men, participated in the driving simulation experiment, ranging in age from 18 to 59. The age and gender distribution of the participant sample was based on crash data for single vehicle run-off-road crashes, and as such, contains a higher proportion of young males.

Filler Segments and Questions. The test conditions were interchanged with filler tiles. The filler tiles were always straight-away segments to help alleviate any simulator discomforts the participant might feel from the curved test segments. These segments also contained various landscaping and objects to help keep the participant interested and believing they were on one long continuous roadway, and not repeating driving segments. During each of the filler segments, one of the following questions would appear in the center of the simulator projector screen: "What was the last sign you passed?"; "What was the color of the last car you passed?" The sole purpose of these questions was to keep the participant's attention on the roadway. The entire drive in one of the test worlds was approximately 15 minutes long. 


\section{METHODOLOGICAL LESSONS LEARNED FROM THE STUDY}

The results of the study are presented in detail in the technical report (Chrysler, Williams, and Meischen, 2005). The discussion here will focus on tips the authors would like to share with other researchers interested in using driving simulation to evaluate the effects of roadway geometrics. We are still in the early stages of using the simulator for traffic engineering studies and may have made some errors. We hope that by sharing our experiences, other researchers can avoid our mistakes.

\section{Simulator Sickness}

In past studies using this facility, we have experienced particularly high rates (up to 75\%) of simulator sickness (Chrysler, Wright, and Williams, 2003). In the current study, only one of our participants became uncomfortable. While not the subject of a specific study, through our experience and best-guess we attribute this reduction to one or more of the following factors:

- Fewer participants over the age of 55 participated in the current study compared to the prior study.

- Removal of the simulator vehicle's windshield increased airflow into the passenger compartment. The removal also equalized the luminance of the front and side views. With the windshield in place, the side screens were viewed through open windows while the front screen was viewed through a tinted windshield. We feel this disparity may have produced a higher luminance scene in peripheral vision and increased the likelihood of sickness.

- The use of filler questions seemed to put participants at ease. The experimenter also engaged in light conversation through an intercom system with the drivers during noncritical sections of the scenarios. This, too, seemed to provide enough distraction so that they were not concentrating so much on the experience of driving the simulator. Obviously, care must be taken so as not to talk to the drivers so much that they become too distracted, but we feel strongly that the normal experimental procedure of testing in silence may contribute to sickness by giving the driver nothing to think about other than their queasy stomach.

- The removal of start and stop conditions compared to earlier studies. The visual "pitch" introduced by braking coupled with the lack of motion from the vehicle seems to particularly induce sickness. By removing any hard braking situations from the scenario we reduced sickness rates.

- Based on prior research, we believed that the presence of curves in the simulator world could cause sickness problems. We did not find this to be the case. We did take care, however, to use large radius curves traversed at high speeds with few roadside objects in peripheral view.

- The use of a telephone pre-screening may have eliminated sickness-prone participants from enrolling in the study. For this study, only a handful of potential participants were rejected based on the pre-screening. This may have been due to the high number of younger drivers contacted. In a current study, focusing more on older drivers, we are rejecting a higher number based on this initial pre-screening. 


\section{Limitations of the Simulator's Visual Rendering}

The advantage of the DriveSafety ${ }^{\mathrm{TM}}$ simulator is its ease of use in constructing new roadway environments and scripting scenarios. Developers are, however, limited to the set of roadway tiles supplied by the vendor. For the current study, we had to contract with an approved programmer to develop the specific roadway geometries required. This process required us to develop extremely detailed drawings of the roadway cross-sections and to go through several iterations of review of the tiles. This design and review process would be required regardless of whether or not an outside programmer was used. We still find it cost-effective to employ outside programmers for occasional enhancements to the simulator library.

Despite the numeric accuracy of the tiles, the appearance of the roadways still was not satisfactory in some regards. Rendering a 3-D image on a flat screen necessitates a sort of "uphill" view to provide visibility of objects in the distance. The perspective of this uphill view seemed to exaggerate the width of the shoulders in the foreground. There was little visual difference between the two- and four-foot shoulder width conditions in our study.

One of the goals of this project internally was to promote collaboration between the traffic engineering and human factors researchers at our Institute. These oddities of the simulator's rendering provided great consternation to our traffic engineer colleagues who complained that it "just didn't look right." Our traffic engineering colleagues also did not like how objects in the distance (e.g., trees, buildings, signs) suddenly appeared on the horizon. This "pop-up" problem may be an artifact of the older software we are using and may not be present in more powerful simulators. While these appearance problems are not to be discounted in a study such as this, for most human factors studies of driver distraction, they may not pose a big problem. Other minor roadway and traffic control features are also wrong in the DriveSafety ${ }^{\mathrm{TM}}$ worlds; things such as some traffic sign designs, pavement markings, and curve radii. All of these minor errors contributed to the traffic engineers rejecting the simulator outright. Clearly, when working with traffic engineers and studying roadway features the verisimilitude of the roadway is very important.

The version of the DriveSafety ${ }^{\mathrm{TM}}$ product that we use is not able to render nighttime scenes. Many of the rural two-lane road crashes we were trying to model occur at night. The compelling simulation of nighttime scenes will be a useful "sales tool" to encourage further use of the simulator to investigate traffic engineering issues.

\section{Data Analysis}

The analysis of data from the simulator is a continual challenge due to the large file sizes. In designing scenarios, the experimenter can decide how frequently to sample the data stream (up to $60 \mathrm{~Hz}$ ). For the current study, we were primarily interested in lane placement and steering inputs. These are both rapidly changing parameters, so we decided to sample at $30 \mathrm{~Hz}$. The resulting SPSS file contains 498,000 cases and is $87 \mathrm{Mb}$. We realize now that we could have likely gotten good estimates of driving performance with a lower sampling rate. One problem with that many data points is that nearly everything is statistically significant even though the practical significance is questionable. 
Another challenge for us in the data analysis has been to determine when exactly the driver crossed the edge line and left the lane. Lane position data are indexed from the centerline of the road. The pavement markings themselves occupy some space on the road (four inches in this case), so that width needs to be accounted for in calculations of lane excursion. For the purposes of this study, we are only flagging a data point as a lane excursion if the right wheel passed over the outside edge of the pavement marking, in effect leaving the paved surface. It is easy to get caught up in these calculations and assume a great deal of accuracy. To the driver of the simulator, however, the edge of the vehicle and the edge of the roadway are very subjective things. Because there is no physical feedback when the vehicle leaves the paved surface, drivers have a hard time judging exactly where the vehicle is positioned laterally in the lane.

\section{CONCLUSIONS}

Driving simulation is one assessment tool available to traffic safety researchers. It has primarily been the domain of human factors specialists studying driver distraction, impairments, and ergonomic issues. The Texas Transportation Institute is attempting to broaden the application of driving simulation to the evaluation of traffic engineering issues such as roadway design and traffic operations. The limitations of our current simulator in terms of verisimilitude to actual roadways somewhat hamper these efforts. Traffic engineers are sticklers for manuals, and often reject simulator research outright if minor roadway features were not recreated perfectly.

Current and future research in our laboratory is having more success collaborating with traffic engineers to evaluate changeable message sign phasing and placement. In these studies, the simulator is used more or less as an elaborate task load and the driver performance measures are reading time and comprehension of signs. Another study is examining traffic signal operations, with particular applications to red-light running. These types of studies utilize the simulator's strengths at providing a real-world driving task in a safe and replicable environment.

\section{REFERENCES}

Chrysler, S.T., Williams, A.A. and Meischen, S. (2005). Traffic Engineering Applications of Driving Simulation. Report Number SWUTC/O5/167142. College Station TX: Southwest University Transportation Center, Texas A\&M University.

Chrysler, S.T., Wright, J., \& Williams A. (2003). A comparison of traffic sign comprehension using static, dynamic and interactive presentation media. Paper presented at the 2nd International Driving Symposium on Human Factors in Driver Assessment, Training, and Vehicle Design. Park City, UT, July 22-25.

Hauer, E. (2000). Lane Width and Safety (unpublished draft). Toronto, Canada: Author. Retrieved October 4, 2004 from http://www.roadwafetyresearch.com

Mounce, J. (2004). Review of the Literature on Two-Lane Rural Roadway Crashes and Countermeasures. College Station TX: Center for Transportation Safety unpublished report, Texas Transportation Institute. 